\title{
Dynamic imaging of allogeneic adipose-derived regenerative cells transplanted in ischemic hind limb of apolipoprotein E mouse model
}

This article was published in the following Dove Press journal:

International Journal of Nanomedicine

21 December 2016

Number of times this article has been viewed

\author{
Yi Zheng',* \\ Jinbao Qin $2, *$ \\ Xin Wang ${ }^{2, *}$ \\ Zhiyou Peng ${ }^{2}$ \\ Peiyong Hou' \\ Xinwu $\mathrm{Lu}^{2,3}$ \\ 'Department of General Surgery, \\ The Fourth Affiliated Hospital of \\ Guangxi Medical University, Guangxi, \\ ${ }^{2}$ Department of Vascular Surgery, \\ School of Medicine, Shanghai \\ Ninth People's Hospital Affiliated \\ to Shanghai JiaoTong University, \\ ${ }^{3}$ Vascular Center of Shanghai \\ JiaoTong University, Shanghai, People's \\ Republic of China \\ *These authors contributed equally \\ to this work
}

Background: Transplantation of allogeneic adipose-derived regenerative cells (ADRCs) is a promising treatment modality for severe ischemic diseases. However, minimal information is available on the in vivo effects, fate, and migration of ADRCs, as well as the mechanisms of their therapeutic angiogenesis.

Materials and methods: In this study, green fluorescent protein-expressing ADRCs (GFPADRCs) were obtained, labeled with acetylated 3-aminopropyltrimethoxysilane (APTS)-coated iron oxide nanoparticles (APTS NPs), and injected into an old apolipoprotein E knockout (ApoE-KO) mouse model with hind limb ischemia. Then, 3.0 T magnetic resonance imaging (MRI) was performed to dynamically trace the role of ADRCs targeting hind limb ischemia in the ApoE-KO mice model.

Results: Labeled cells were visualized as large hypointense spots in ischemic muscles by serial 3.0 T MRI scans during a 4-week follow-up. The presence of labeled GFP-ADRCs was confirmed by Prussian blue staining and fluorescence microscopy on postmortem specimens.

Conclusion: This study showed that allogeneic ADRCs offer great potential application for therapeutic angiogenesis in severe ischemic disease based on the efficacy and feasibility of ADRC transplantation and on the available amounts of tissue.

Keywords: allogeneic adipose-derived stem cells, cell tracking, APTS nanoparticles, hind limb ischemia, ApoE knockout mouse

\section{Introduction}

Peripheral arterial occlusive disease (PAD) caused by atherosclerosis is becoming a critical public health problem in developed and developing countries. ${ }^{1,2}$ Hind limb ulceration and gangrene caused by progression of tissue hypoperfusion occur in the late stages of total occlusive peripheral vascular disease. Unfortunately, amputation is needed in more than a third of patients suffering from very severe PAD. ${ }^{3,4}$ Rapid and efficient revascularization of ischemic limb is significant to restore the function of lower limbs. ${ }^{5}$

Stem cells demonstrate tremendous potential to stimulate differentiation of various tissues, such as ischemic lower limb, ${ }^{6}$ cardiac muscle, ${ }^{7}$ nerve, ${ }^{8}$ and bones. ${ }^{9}$ Recent reports have shown that adipose tissues could supply abundant adipose-derived regenerative cells (ADRCs), which are pluripotent stem cells that can self-renew and differentiate into various cell types and can regenerate damaged tissues and organs. ${ }^{10-12}$ Thus, ADRCs offer great potential applications in regenerative medicine. However, the mechanism by which implanted ADRCs regenerate angiogenesis in ischemic tissues is unclear.
Dovepress
International Journal of Nanomedicine 20|7:|2 6|-7|

61

(c) (1) \& () 2017 Zheng et al. This work is published and licensed by Dove Medical Press Limited. The full terms of this license are available at https://www.dovepress.com/terms.php (c) hereby accept the Terms. Non-commercial uses of the work are permitted without any further permission from Dove Medical Press Limited, provided the work is properly attributed. For permission hereby accept the Terms. Non-commercial uses of the work are permitted without any further permission from Dove Mer.
for commercial use of this work, please see paragraphs 4.2 and 5 of our Terms (https://www.doveperss.com/terms.php). 
To evaluate the effects of stem cell-based therapies that are used to repair ischemic lower limbs, we must noninvasively detect the location, migration, and long-term fate of implanted cells. ${ }^{13,14}$ This goal can be achieved through magnetic resonance imaging (MRI) of the transplanted cells labeled with magnetically visible nanoparticles. ${ }^{15,16}$ The superiority of MRI in tracking and monitoring transplanted stem cells has been established using different cell types, such as bone mesenchymal stem cells (BMSCs), peripheral blood stem cells, and embryonic stem cells. ${ }^{17}$ Superparamagnetic iron oxide (SPIO) nanoparticles are the most sensitive MRI contrast agents used in cell labeling. They are safe and biodegradable, and they do not affect the proliferation and differentiation capacity of implanted cells in vitro and in vivo. ${ }^{18-20}$ However, minimal information is available on the outcome and therapeutic capacity of ADRCs labeled with magnetic 3-aminopropyltrimethoxysilane (APTS)-coated iron oxide nanoparticles (APTS NPs) and in the absence of transfection agents.

Thus, the present study aimed to test the feasibility and efficacy of ADRCs labeled with APTS NPs and to evaluate cellular imaging of cell viability, distribution, and fate of labeled ADRCs transplanted into apolipoprotein E knockout (ApoE-KO) mouse model with ischemic limbs. We specially examined whether the transplanted ADRCs could regenerate collateral vessel formation over a long period of time.

\section{Materials and methods Isolation of mouse ADRCs}

ADRC cultures were prepared according to a reported protocol. ${ }^{21}$ Briefly, ADRCs were obtained from inguinal fat pads of green fluorescent protein (GFP)-transgenic mice with C57BL/6J background ( $\mathrm{n}=30)$ under sterile conditions as described previously. ${ }^{21}$ Dulbecco's Modified Eagle's Medium (DMEM) containing 10\% fetal bovine serum and antibiotic/antimycotic solution (Thermo Fisher, Carlsbad, CA, USA) was used.

On day 7 , the expression profile of $\mathrm{P} 3$ attaching cell surface marker was evaluated by fluorescence activated cell sorting (FACS). Cells $\left(5 \times 10^{5}\right)$ were incubated for 30 minutes at $4{ }^{\circ} \mathrm{C}$ with monoclonal antibody specific for mouse cluster of differentiation (PECAM-1 or CD31, CD34, CD90, CD105, and MHC-II (Biomedical Technology Inc.) or with unstained control for FACS Calibur analysis using FlowJo software (Becton, Dickinson and Company, Franklin Lakes, NJ, USA).

\section{ADRC culture and labeling}

After 24 hours of incubation, the ADRCs were labeled with APTS NPs $(25 \mu \mathrm{g} / \mathrm{mL}$, a protocol with known safety and efficacy $^{22}$ ) for 20 hours. The control ADRCs were incubated with DMEM. The labeling efficiency of ADRCs was determined by Prussian blue staining. After 1, 3, 5, and 7 days of culturing, cell viabilities were assessed by Cell Counting Kit-8 (CCK8; Dojindo Molecular Technologies, Japan). Absorbance measurements of the labeled ADRCs were compared with those of unlabeled ADRCs and were analyzed thrice. Moreover, the viability and apoptosis of ADRCs after labeling with APTS NPs were determined by 7 aminoactinomycin $\mathrm{D}$ (7AAD) and Annexin V staining and then analyzed by flow cytometry (BD Biosciences, Rockville, MD, USA).

\section{Cell differentiation}

The capability of APTS NP-labeled and -unlabeled ADRCs to differentiate into adipocytes and osteoblasts was investigated as follows. Approximately $4 \times 10^{5} \mathrm{ADRCs}$ per well were plated in a six-well plate and then cultured for 48 hours. Three cycles of induction with adipogenic and osteoblast induction medium (Cambrex) were added to the six-well plate to stimulate differentiation of adipocytes and osteoblasts. After 3 weeks, the medium was gently suctioned, and the cells were fixed in 4\% formalin and stained with Oil Red $\mathrm{O}$ and alizarin red. Accordingly, expressions of the osteogenic representative gene (Runx2) and the adipogenic representative gene (PPARr) were analyzed using quantitative reverse transcriptase polymerase chain reaction (qRT-PCR). The cDNAs were analyzed using the following primers: Runx2, 5' GGA CTG TGG TTA CCG TCA T 3' and 5' GGA GGA TTT GTG AAG ACT GTT 3' and PPARr, 5' CAC TCG CAT TCC TTT GAC AT 3' and 5' TTG ATC GCA CTT TGG TAT TCT 3'.

\section{ELISA}

To investigate the role of paracrine factors regulated upon ADRC activation, we obtained conditioned medium from the labeled and unlabeled ADRCs at days 1, 2, and 3. Concentrations of mouse bFGF, SDF-1, VEGF, and HGF proteins (R\&D Systems, Minneapolis, MN, USA) in the conditioned media were tested by enzyme linked immunosorbent assay (ELISA) following the manufacturer's instruction.

\section{In vivo cell delivery and MRI}

All procedures were performed on 30- to 40-week-old female ApoE-KO mice (Shanghai Biomodel Organism Science \& Technology Development Co. Ltd., Shanghai, People's Republic of China; $n=18$ ) according to the guidelines prescribed by the Animal Experiment and Care Committee of Shanghai JiaoTong University, School of Medicine. All animal protocols were approved by the Animal Experiment and Care Committee of Shanghai JiaoTong University, School of Medicine. The mice were anesthetized via isoflurane $(2 \%-3 \%)$ inhalation. To investigate the relationship of persistence of MRI signal 
to cell survival and engraftment, we injected $\sim 1 \times 10^{6}$ labeled and unlabeled ADRCs in $100 \mu \mathrm{L}$ of phosphate buffer saline (PBS) into the right ischemic hind limbs of the mice. The $3.0 \mathrm{~T}$ MRI scans were obtained $1,7,14,21$, and 28 days after cell engraftment. At least three consecutive short-axis slices were obtained to confirm the area of cell injection. MR images were analyzed by ImageJ software (Bethesda, MD, USA). Signal intensity in the hind limb was also determined. Laser Doppler blood perfusion imagers (Omegaflow FLO-CI; Omegawave, Portland, OR, USA) were performed to measure the cutaneous blood flow at 4 weeks after transplantation.

\section{Histology and immunohistochemistry}

The mice were euthanized 4 weeks after ADRC transplantation. Both hind limbs were embedded in paraffin and cut into $5 \mu \mathrm{m}$ sections. Hematoxylin and eosin (H\&E) staining and Prussian blue staining were performed to identify the fate of the transplanted ADRCs. For iron particle staining, sections were incubated with Perls reagent in the dark for 30 minutes and then counterstained with fast red.

For histological examination via immunofluorescence, $5 \mu \mathrm{m}$ frozen sections were cut. For immunostaining, sections were incubated with primary antibodies, rabbit anti-mouse CD31 (Santa Cruz Biotechnology Inc., Santa Cruz, CA, USA), followed by incubation with secondary antibody, APC-conjugated mouse anti-mouse IgG (BD Pharmingen, NY, USA), and Alex 555-conjugated goat anti-rabbit IgG (Thermo Fisher), and then counterstained with 4',6-diamidino-2-phenylindole (DAPI).

Lungs, liver, and spleen were collected, fixed, and embedded in paraffin. Serial sections were stained with H\&E and iron staining to detect iron particles for evaluation of morphological changes. Controls were also established in the unlabeled groups.

\section{Statistics}

Data are expressed as mean \pm standard error of the mean. Statistical significance was evaluated using unpaired Student's $t$-test for comparison between two groups. Values of $P<0.05$ (*) denoted statistical significance.

\section{Results}

\section{Characterization of cultured ADRCs}

P3 ADRCs are fibroblast-like cells in terms of shape and were culture expandable every other day (Figure 1A). The ADRCs obtained from GFP-transgenic mice continuously expressed Luc-eGFP reporter gene, which are shown green in Figure 1B. In addition, the Luc-eGFP activity did not change significantly in in vitro ADRC culture (data not shown).
FACS analysis showed that P3 ADRCs were positive for stem cell marker CD90 (59.7\% $\pm 0.6 \%)$ and CD105 (76.5\% $\pm 0.8 \%$ ) but negative for $1.3 \% \pm 0.1 \% \mathrm{CD} 31$ (a marker of endothelial cells), $1.2 \% \pm 0.1 \%$ CD34 (hematopoietic and endothelial progenitor cell marker) cells, and $0.9 \% \pm 0.1 \%$ MHC-II (Figure 1).

\section{ADRC labeling and cytotoxicity}

ADRCs were labeled with APTS NPs and tested for labeling efficiency and cell viability. Microscopic observation revealed APTS NPs in the cytoplasm. In Figure 2A, 100\% of the ADRCs incorporated APTS NPs and are stained blue after 24 hours of culturing. The unlabeled ADRCs showed no blue staining particles in the cytoplasm (Figure 2B). CCK8 assay showed that viability of ADRCs remained at $>95 \%$ after APTS NP labeling for at least 7 days (Figure 3A), and no increase in apoptosis was observed on day 3 as shown by FACS analysis (Figure 3B). Moreover, no difference in the Annexin rates was observed between APTS NP-labeled (Figure 3C) and APTS NP-unlabeled ADRCs (Figure 3B).

Iron labeling of ADRCs with $25 \mu \mathrm{g} / \mathrm{mL}$ APTS NPs did not increase cell necrosis nor apoptosis $(P=0.530)$ as revealed by flow cytometry (Figure 3B and C). Therefore, $25 \mu \mathrm{g} / \mathrm{mL}$ APTS NPs was suitable for the in vivo experiments.

\section{Cell differentiation}

ADRCs could differentiate into mature adipocytes and osteoblasts in appropriate concentrations of adipocytes and osteoblasts differentiation media. In in vitro culture experiments, differentiation of APTS NP-labeled and -unlabeled ADRCs into adipocyte lineages was identified through microscopic observation of intracellular red lipid droplets and positive reaction to Oil Red O (Figure 4A and B) and qRT-PCR of Runx2 (Figure 4C). Moreover, differentiation of APTS NP-labeled and -unlabeled ADRCs into the osteoblast was confirmed through alizarin red staining, and the result demonstrated that ADRCs could differentiate into calcium nodules (Figure 4D and E). The qRT-PCR of the relative adipocytes and osteoblasts gene expression levels in APTS NP-labeled groups showed no significant difference in the transcript levels of Runx2 (Figure 4C) and PPARr (Figure 4F) compared with the ADRCs control groups.

\section{Angiogenic cytokine production by ADRCs}

To prove that ADRCs secrete a certain amount of paracrine factors, including bFGF, SDF-1, VEGF, and HGF proteins, the conditioned culture medium analyzed by using ELISA. Concentrations of bFGF, SDF-1, VEGF, and HGF 

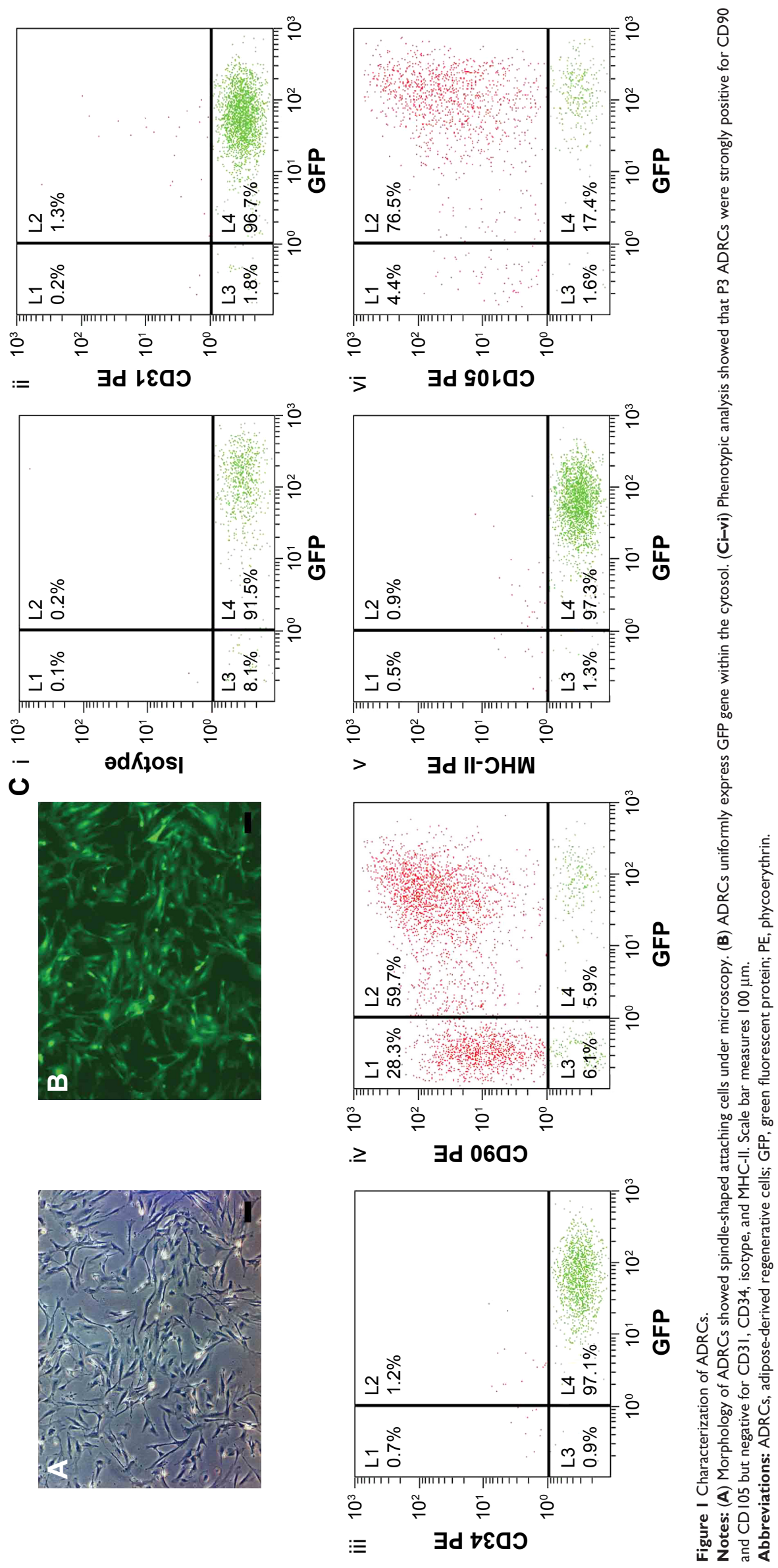

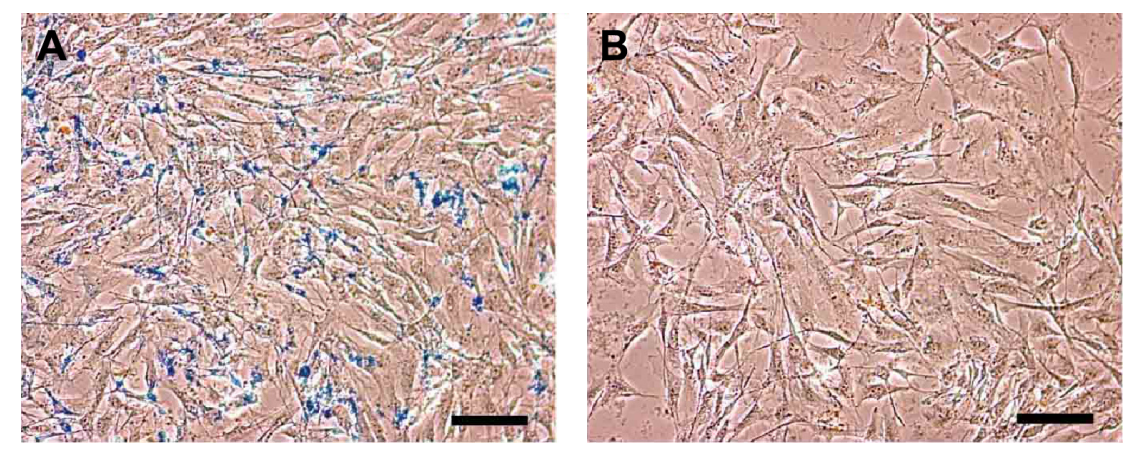

Figure 2 ADRCs labeled with APTS NPs.

Notes: (A) Prussian blue staining showed that $\sim 100 \%$ of the ADRCs incorporated APTS NPs and are stained blue after 24 hours of culturing. (B) The unlabeled ADRCs showed no blue staining particles in the cytoplasm. Scale bar measures $100 \mu \mathrm{m}$.

Abbreviations: ADRCs, adipose-derived regenerative cells; APTS NPs, 3-aminopropyltrimethoxysilane-coated iron oxide nanoparticles.

in conditioned medium showed no significant difference between the labeled and unlabeled ADRCs (Figure 5).

\section{In vivo tracking of ADRCs}

The mice were scanned on days 1, 7, 14, 21, and 28 to investigate the migration of the engrafted cells in hind limb. The 3.0 T MR images are shown in Figure 6. ADRCs could be clearly identified in the adductor muscles, and their migration could be monitored for at least 4 weeks. The hypointensities ("black spots") were found in the region of ADRC injection in all the mice transplanted with APTS NP-labeled ADRCs (Figure 6A). In the cross section of the hind limb of the ApoE-KO mouse models 1 week after treatment, the site of injection exhibited wide hypointensities, which extended beyond the actual location of the labeled ADRCs ("blooming effect"), in addition, the hypointensities decreased with time. Control-unlabeled ADRCs or saline-injected groups showed no hypointensities in their muscles as revealed by MRI (Figure 6A). Four weeks after cell transplantation, black spots could still be observed under MRI (Figure 6A). Control animals injected with unlabeled cells expectedly showed no hypointensities MR signals, as expected. The data of the MRI signal values in the APTS NP-labeled groups were significantly $(P<0.05)$ lower compared with the unlabeled ADRCs groups and gradually increased to normal levels at 4 weeks (Figure 6B). Representative color laser Doppler images of superficial blood flow in lower ischemic limbs before and after treatment are shown in Figure S1. We found that the superficial blood flow was higher after the APTS NP-labeled ADRC treatment than before the treatment (Figure S1).

\section{Location of implanted ADRCs}

We examined whether transplanted ADRCs could survive and differentiate into endothelial cells (ECs) in vivo. Immunofluorescence staining showed that some of the GFP-positive cells were retained near vascular structures and capillaries stained with CD31 (Figure 7A-C) and some of the ADRCs were also GFP positive. Quantitative analysis demonstrated that capillary density in ischemic hind limb muscles was significantly greater in the APTS NP-labeled and -unlabeled ADRCs groups than in the control groups $(P<0.05$ versus control; Figure 7D). Our findings indicated that the transplanted
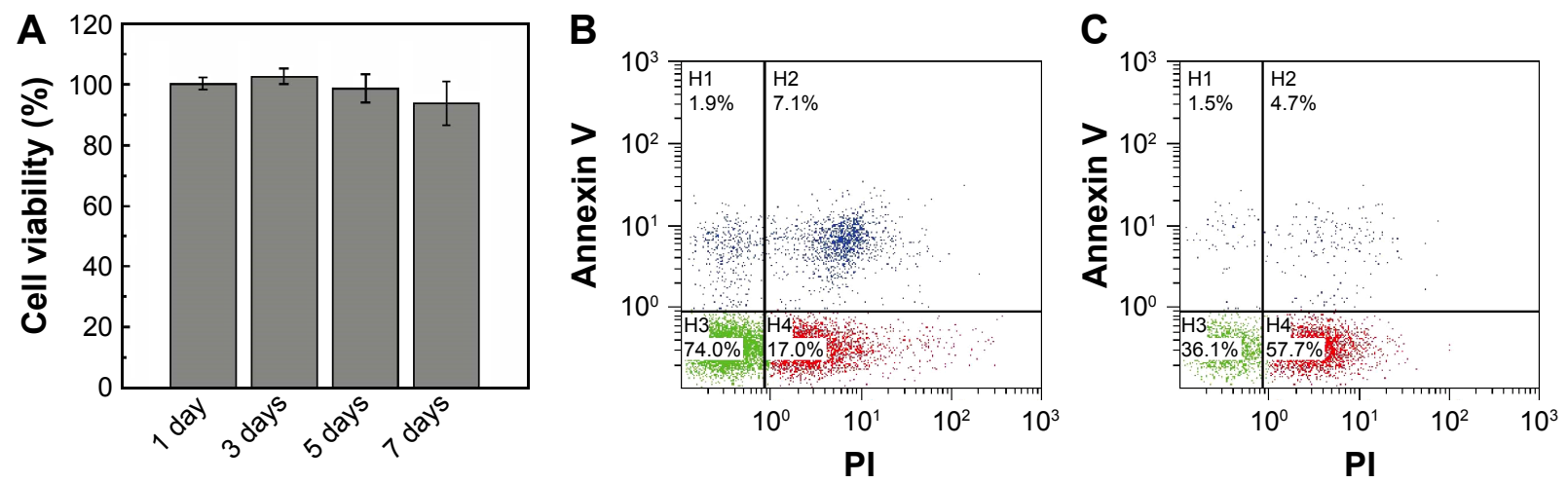

Figure 3 Cell viability and apoptosis of ADRCs.

Notes: (A) Viability was assessed by CCK8 at I, 3, 5, and 7 days. (B) Annexin V/PI rate of APTS NP-labeled ADRCs. (C) Annexin V/PI rate of non-APTS NPs labeled ADRCs.

Abbreviations: ADRCs, adipose-derived regenerative cells; APTS NP, 3-aminopropyltrimethoxysilane-coated iron oxide nanoparticle. 

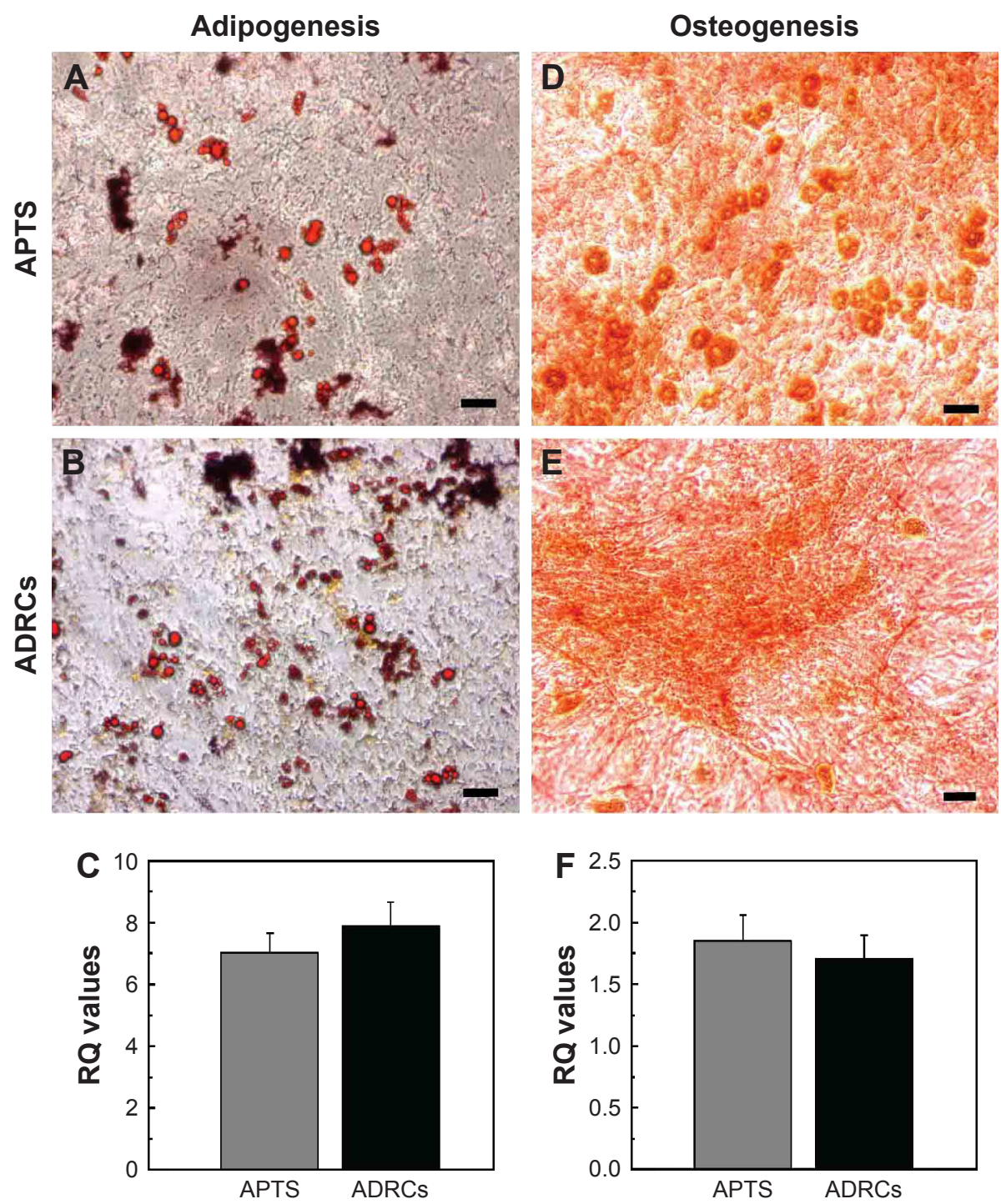

Figure 4 Multidifferentiation assays of APTS NP-labeled and -unlabeled ADRCs.

Notes: Adipocyte differentiation potential of the APTS NP-labeled ADRCs (A) and APTS NP-unlabeled ADRCs (B) was assessed by oil red O staining. (C) qRT-PCR of Runx2. Osteocytes' differentiation potential of the APTS NP-labeled ADRCs (D) and APTS NP-unlabeled ADRCs (E) was assessed by alizarin S staining. (F) qRT-PCR of PPARr. Scale bar measures $100 \mu \mathrm{m}$.

Abbreviations: ADRCs, adipose-derived regenerative cells; APTS NP, 3-aminopropyltrimethoxysilane-coated iron oxide nanoparticles; qRT-PCR, quantitative reverse transcriptase polymerase chain reaction; $R Q$, relative quality.

ADRCs already differentiated into the endothelial cells and possibly contributed to vascular formation to a certain extent. Furthermore, no tumors were formed in transplanted mouse after at least 30 days (data not shown).

\section{Histological analysis}

ADRCs were particularly visualized in the ischemic zone of the hind limb after Prussian blue staining (Figure 8). No Prussian blue-positive cells were localized in the ischemic hind limb of the unlabeled group (Figure 8).

To further investigate whether the remaining APTS NPs could accumulate in different target organs, the liver, lungs, and spleens of the mouse models were collected and then stained them with H\&E and Prussian blue staining. Prussian blue-positive staining, which represents iron, presumably from APTS NP labeling of the ADRCs, was detected in the liver, spleen, and lung (Figure 8). The results showed that APTS NPs did not accumulate in the lungs of any of the mouse models. However, a small amount of APTS NPs was found in livers and spleens from APTS NP groups of mouse models.

\section{Discussion}

The present study found that 1) P3 ADRCs expressed mesenchymal stem cell markers and were negative for endothelial lineage markers (CD31) in vitro. 2) P3 ADRCs could be efficiently and safely labeled with APTS NPs. 3) Direct local implantation of APTS NP-labeled ADRCs into ischemic 


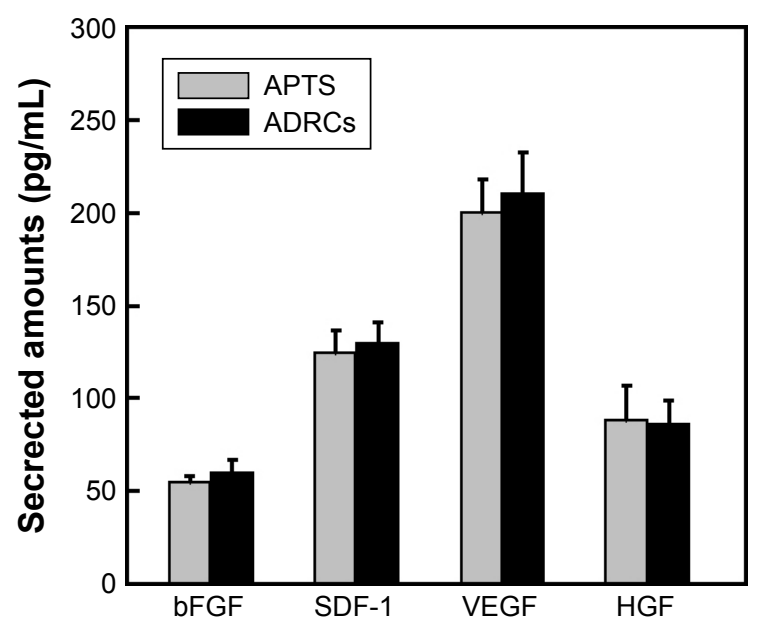

Figure 5 ELISA of APTS NP-labeled and -unlabeled ADRCs.

Notes: ELISA showed that APTS NP-labeled and -unlabeled ADRCs secrete a certain amount of paracrine factors, including bFGF, SDF-I, VEGF, and HGF proteins. There was no significant difference between the labeled and unlabeled ADRCs.

Abbreviations: ADRCs, adipose-derived regenerative cells; APTS NP, 3aminopropyltrimethoxysilane-coated iron oxide nanoparticle.

hind limb muscles could be monitored by 3.0 T MRI and can improve neovascularization.

Adipose tissues comprise two main cell types, namely, adipocytes and ADRCs. ${ }^{23}$ Studies have proven that ADRCs contain multipotent mesenchymal stem cells that can differentiate into various cell types. Thus, they offer great potential applications in regenerative medicine. ${ }^{24} \mathrm{MRI}$ is a highly superior technique in tracking and monitoring transplanted stem cells labeled with contrast agents (such as SPIO). ${ }^{25}$ However, Kostura et $\mathrm{al}^{26}$ reported that chondrogenic differentiation of BMSCs but not adipogenesis could be impaired. In this study, APTS NPs were used instead of SPIO and found no significant difference in the differentiation potential between APTS-labeled and -unlabeled ADRCs. APTS NPs do not require addition of some transfection reagents to enhance the labeling efficiency compared with SPIO, which may inhibit stem cell differentiation ability into chondrocytes. The procedure used to label ADRCs with APTS NPs without poly-L-lysine (PLL) is highly efficient and safe, as well as more practical than that used in a previous study. ${ }^{27}$ We used a relatively low APTS NP concentration $(25 \mu \mathrm{g} / \mathrm{mL})$ as previously used in several trials, which have shown clear MRI visualization of ADRCs without altering the viability, apoptotic indices, survival, migration, and differentiation compared with those of their unlabeled counterparts.

Long-term observation of APTS NP-labeled ADRCs might be limited to a certain extent because of APTS dilution
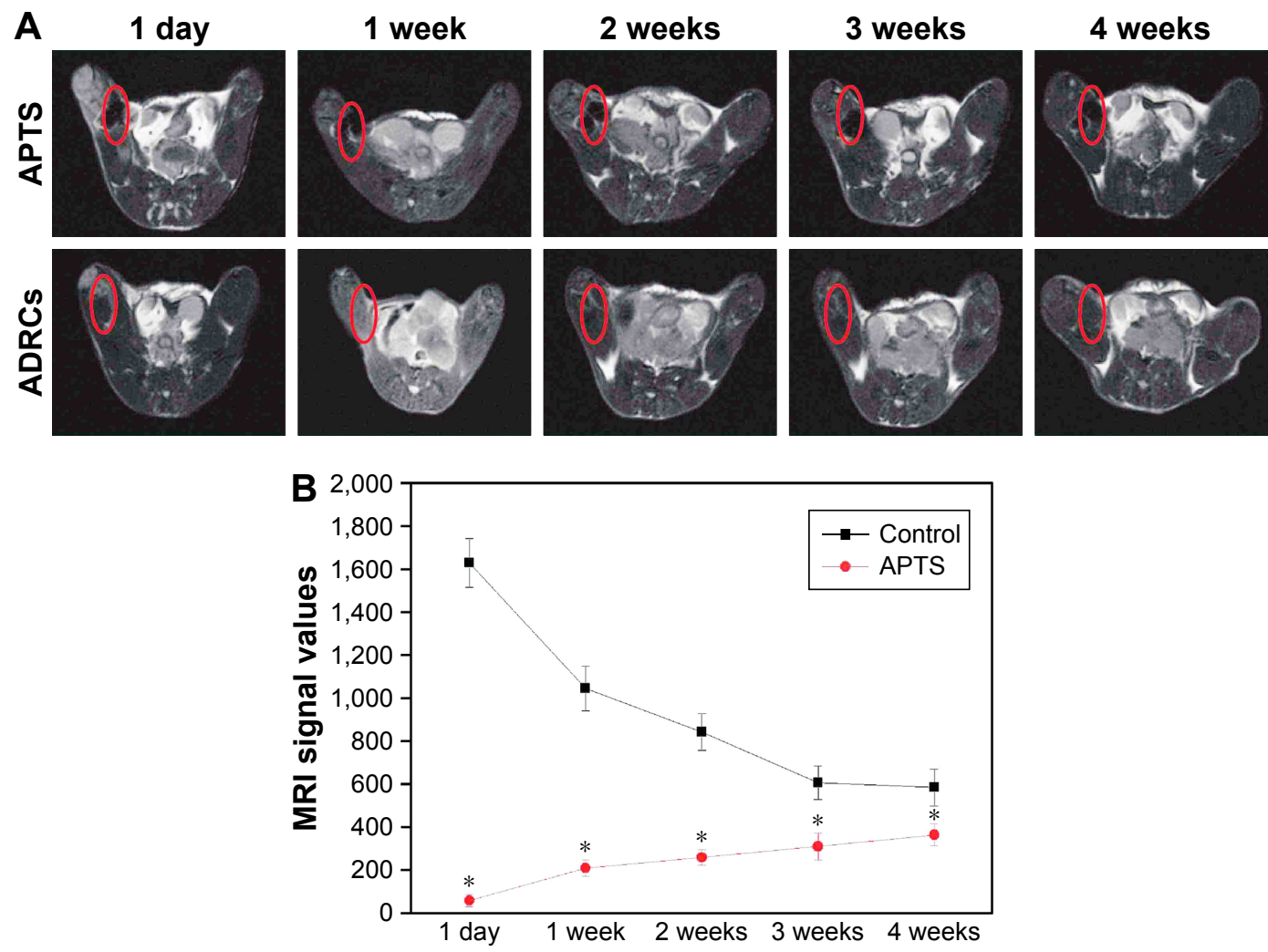

Figure 6 In vivo tracking of APTS NP-labeled and -unlabeled ADRCs by 3.0 T MRI.

Notes: (A) Mice were scanned at days I, 7, 14, 21, and 28 to investigate the migration of the engrafted cells in the hind limb ischemia. (B) Quantitative analysis of MRI signal values revealed that APTS NP groups exhibited significant difference compared to ADRC groups $(* P<0.05)$.

Abbreviations: ADRCs, adipose-derived regenerative cells; APTS NP, 3-aminopropyltrimethoxysilane-coated iron oxide nanoparticles; MRI, magnetic resonance imaging. 

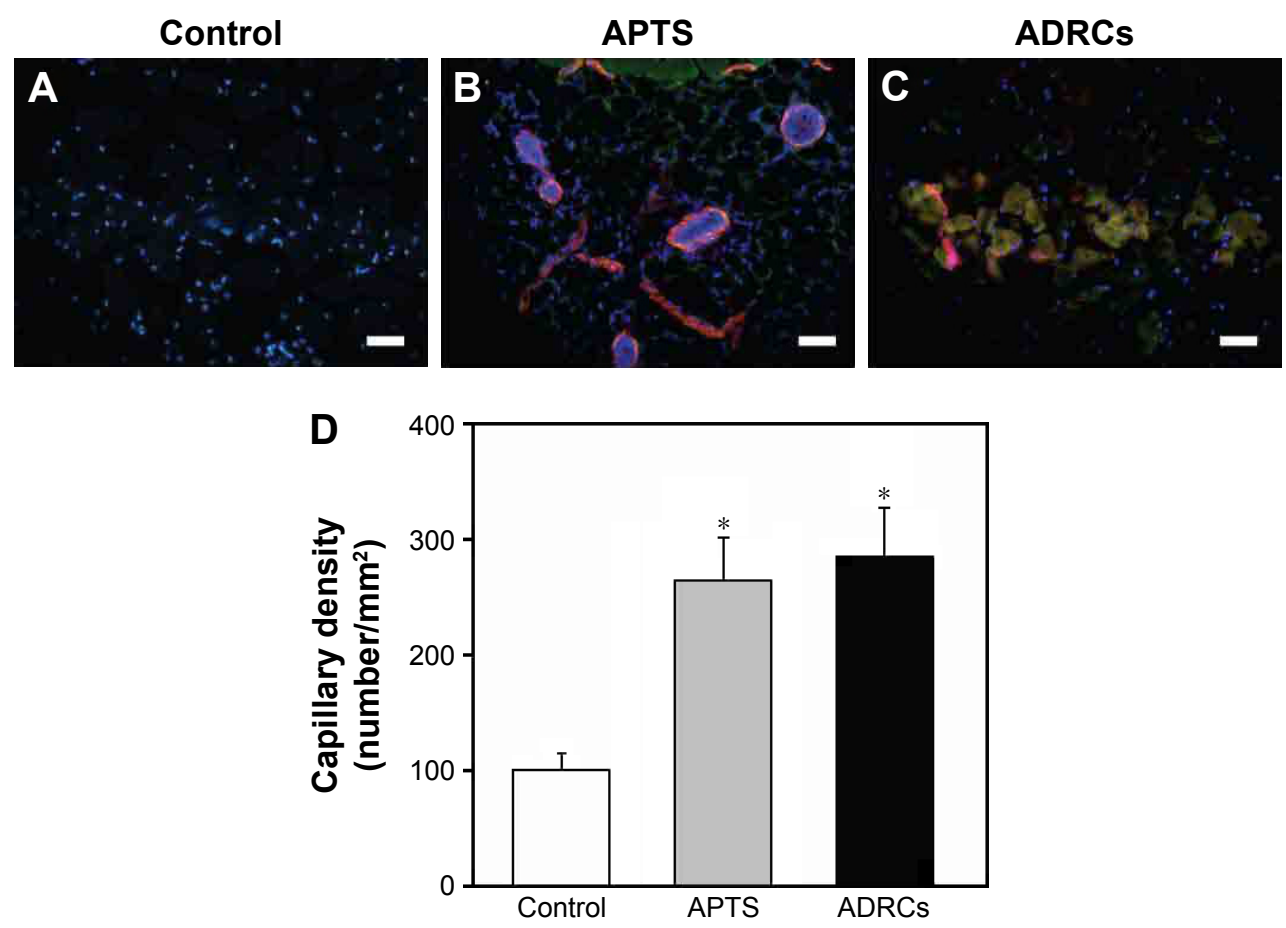

Figure 7 Immunofluorescent staining of the ischemic muscles after ADRC transplantation.

Notes: Representative immunohistochemistry staining of the microvessel densities for endothelial cell markers CD3I in the control groups (A), APTS groups (B), and ADRC groups (C). (D) Statistical analysis of the microvessel densities revealed that APTS NP-labeled and -unlabeled ADRC groups exhibited significant difference compared to the control groups $(* P<0.05)$. Scale bar measures $100 \mu \mathrm{m}$.

Abbreviations: ADRCs, adipose-derived regenerative cells; APTS NP, 3-aminopropyltrimethoxysilane-coated iron oxide nanoparticle.
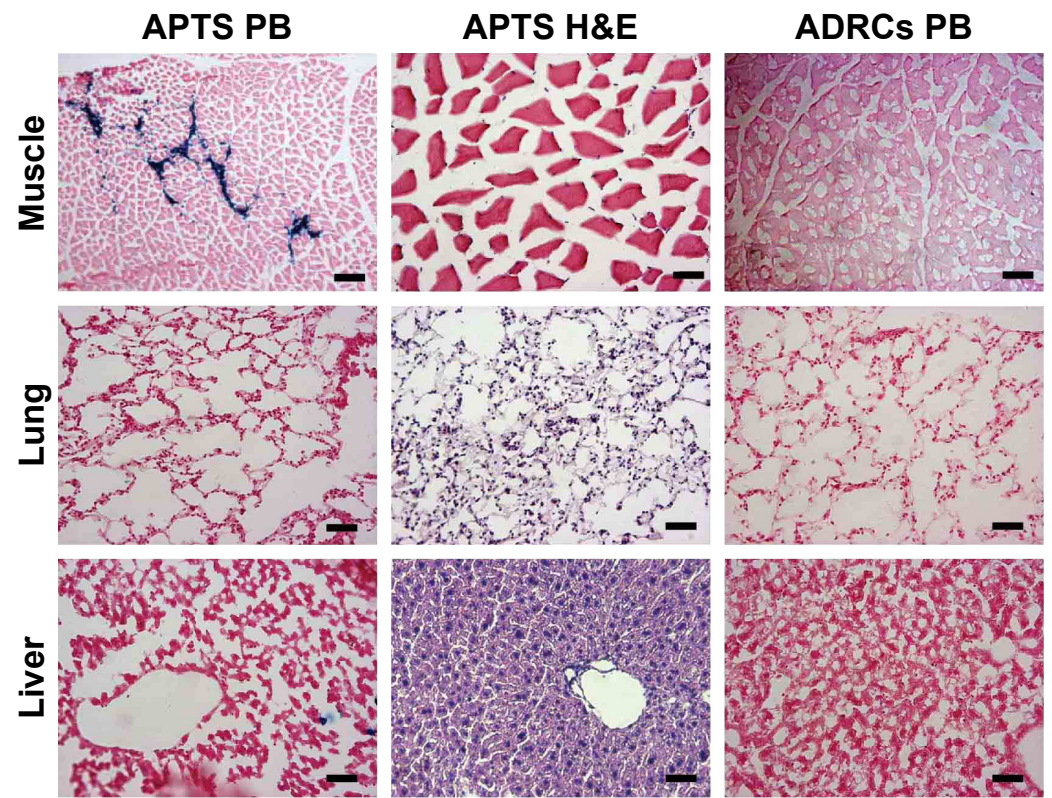

ADRCs H\&E
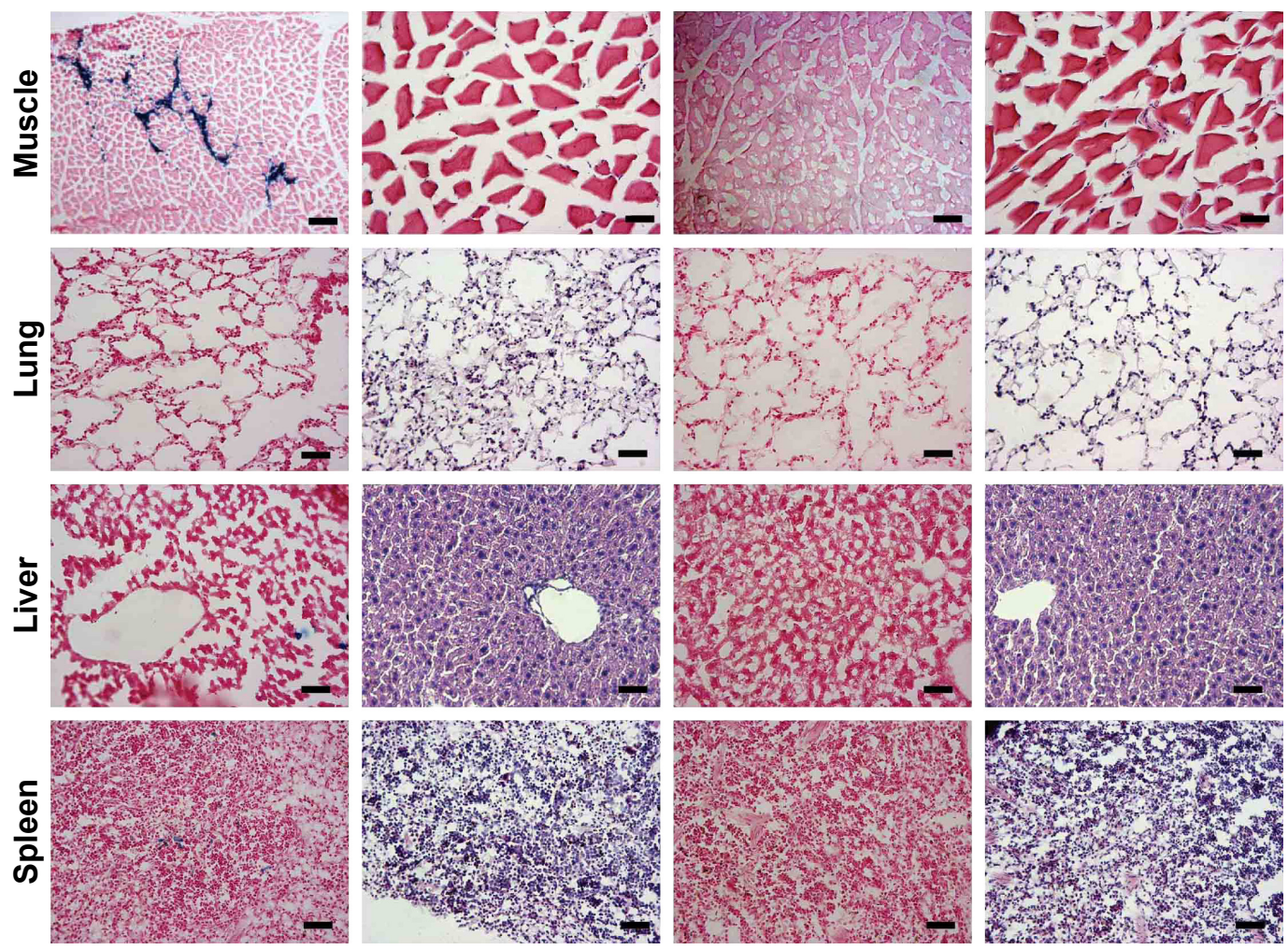

Figure 8 Immunohistochemistry staining of the muscles and the organs.

Notes: PB staining and H\&E staining of the serial sections of the muscles, lungs, liver, and spleen. Scale bar measures $100 \mu \mathrm{m}$.

Abbreviations: ADRCs, adipose-derived regenerative cells; APTS, 3-aminopropyltrimethoxysilane; H\&E, hematoxylin and eosin; PB, Prussian blue. 
as ADRCs divided. To date, some studies have proven that MRI signal originating from surviving APTS NP-labeled cells may be overestimated because of phagocytosis by macrophages and that extracellular iron particles contribute to MRI signal. ${ }^{28,29}$ To address these problems, we compared the use of GFP reporter gene marker and APTS NPs in labeling ADRCs in vitro and in vivo. After double labeling, the fate of implanted ADRCs could be simultaneously monitored by MRI and immunofluorescent microscope. It was found that the relative APTS NP-labeled ADRCs can be detected for at least 4 weeks by MRI and immunofluorescent microscope.

Limited information is available on the underlying mechanisms of allogeneic ADRCs in therapeutic angiogenesis. Boden et $\mathrm{al}^{30}$ showed that ADRC transplantation improved angiogenesis resulting from secretion of angiogenic factors. Moreover, this study demonstrated that ADRC implantation significantly augmented angiogenesis not only by endothelial differentiation (VEGF, HGF, and bFGF) but also by chemokines, such as SDF-1. SDF-1 may play an important role in the ADRC-regenerated angiogenesis to a certain extent by facilitating mobilization of ADRCs.

\section{Conclusion}

Our study demonstrated that APTS NPs are safe and biodegradable and they do not affect the proliferation and differentiation capacity of in vitro and in vivo of cells transplanted into the ischemic hind limb. The results of this study will provide a new source of seed cells for severe ischemic diseases and bring new insights into regenerative therapies.

\section{Acknowledgments}

This research is financially supported by the National Natural Science Foundation of China (81370423, 81570432, 81601621, and 81670440) and the Natural Science Foundation of Shanghai Science and Technology Committee (grant nos 134119a2100 and 20124Y132) and is also sponsored by Shanghai Sailing Program (grant no 15YF1406900) and Shanghai JiaoTong University School of Medicine Doctoral Innovation foundation (BXJ201428).

\section{Disclosure}

The authors report no conflicts of interest in this work.

\section{References}

1. Stoner MC, Calligaro KD, Chaer RA, et al. Reporting standards of the Society for Vascular Surgery for endovascular treatment of chronic lower extremity peripheral artery disease. J Vasc Surg. 2016;64(1):e1-e21.

2. Hamur H, Onk OA, Vuruskan E, et al. Determinants of chronic total occlusion in patients with peripheral arterial occlusive disease. Angiology. Epub 2016 Apr 7.
3. Vallabhaneni R, Kalbaugh CA, Kouri A, Farber MA, Marston WA Current accepted hemodynamic criteria for critical limb ischemia do not accurately stratify patients at high risk for limb loss. J Vasc Surg. 2016; 63(1):105-112.

4. Mutirangura $P$, Ruangsetakit C, Wongwanit C, et al. Enhancing limb salvage by non-mobilized peripheral blood angiogenic cell precursors therapy in patients with critical limb ischemia. J Med Assoc Thai. 2009; 92(3):320-327.

5. Dominguez A 3rd, Bahadorani J, Reeves R, Mahmud E, Patel M. Endovascular therapy for critical limb ischemia. Expert Rev Cardiovasc Ther. 2015;13(4):429-444.

6. Mohamed Omer S, Krishna SM, Li J, Moxon JV, Nsengiyumva V, Golledge J. The efficacy of extraembryonic stem cells in improving blood flow within animal models of lower limb ischaemia. Heart. 2016; 102(1):69-74.

7. Jiang Q, Yu T, Huang K, Lu J, Zhang H, Hu S. Remote ischemic postconditioning ameliorates the mesenchymal stem cells engraftment in reperfused myocardium. PLoS One. 2016;11(1):e0146074.

8. Wang Y, Liu H, Ma H. Intrathecally transplanting mesenchymal stem cells (MSCs) activates ERK1/2 in spinal cords of ischemia-reperfusion injury rats and improves nerve function. Med Sci Monit. 2016;22: $1472-1479$.

9. Ono N, Kronenberg HM. Bone repair and stem cells. Curr Opin Genet Dev. 2016;40:103-107.

10. Balducci L, Alessandri G. Isolation, expansion, and immortalization of human adipose-derived mesenchymal stromal cells from biopsies and liposuction specimens. Methods Mol Biol. 2016;1416:259-274.

11. Legzdina D, Romanauska A, Nikulshin S, Kozlovska T, Berzins U. Characterization of senescence of culture-expanded human adipose-derived mesenchymal stem cells. Int J Stem Cells. 2016;9(1):124-136.

12. Kim JH, Park Y, Jung Y, Kim SH, Kim SH. Combinatorial therapy with three-dimensionally cultured adipose-derived stromal cells and selfassembling peptides to enhance angiogenesis and preserve cardiac function in infarcted hearts. J Tissue Eng Regen Med. Epub 2016 Jun 3.

13. Wolfs E, Verfaillie CM, Van Laere K, Deroose CM. Radiolabeling strategies for radionuclide imaging of stem cells. Stem Cell Rev. 2015; 11(2):254-274.

14. Rodriguez-Porcel M. In vivo imaging and monitoring of transplanted stem cells: clinical applications. Curr Cardiol Rep. 2010;12(1):51-58.

15. Ariza de Schellenberger A, Kratz H, Farr TD, et al. Labeling of mesenchymal stem cells for MRI with single-cell sensitivity. Int J Nanomedicine. 2016;11:1517-1535.

16. Elhami E, Dietz B, Xiang B, et al. Assessment of three techniques for delivering stem cells to the heart using PET and MR imaging. EJNMMI Res. 2013;3(1):72.

17. Kalber TL, Ordidge KL, Southern P, et al. Hyperthermia treatment of tumors by mesenchymal stem cell-delivered superparamagnetic iron oxide nanoparticles. Int J Nanomedicine. 2016;11:1973-1983.

18. Qin J, Li K, Peng C, et al. MRI of iron oxide nanoparticle-labeled ADSCs in a model of hindlimb ischemia. Biomaterials. 2013;34(21): 4914-4925.

19. Busato A, Bonafede R, Bontempi $\mathrm{P}$, et al. Magnetic resonance imaging of ultrasmall superparamagnetic iron oxide-labeled exosomes from stem cells: a new method to obtain labeled exosomes. Int J Nanomedicine. 2016;11:2481-2490.

20. Skelton RJ, Khoja S, Almeida S, et al. Magnetic resonance imaging of iron oxide-labeled human embryonic stem cell-derived cardiac progenitors. Stem Cells Transl Med. 2016;5(1):67-74.

21. Qin JB, Li KA, Li XX, et al. Long-term MRI tracking of dual-labeled adipose-derived stem cells homing into mouse carotid artery injury. Int J Nanomedicine. 2012;7:5191-5203

22. Li XX, Li KA, Qin JB, et al. In vivo MRI tracking of iron oxide nanoparticle-labeled human mesenchymal stem cells in limb ischemia. Int J Nanomedicine. 2013;8:1063-1073.

23. Comella K, Parcero J, Bansal H, et al. Effects of the intramyocardial implantation of stromal vascular fraction in patients with chronic ischemic cardiomyopathy. J Transl Med. 2016;14(1):158. 
24. Guo LZ, Kim TH, Han S, Kim SW. Angio-vasculogenic properties of endothelial-induced mesenchymal stem cells derived from human adipose tissue. Circ J. 2016;80(4):998-1007.

25. Patsula V, Kosinova L, Lovric M, et al. Superparamagnetic Fe3O4 nanoparticles: synthesis by thermal decomposition of iron(III) glucuronate and application in magnetic resonance imaging. ACS Appl Mater Interfaces. 2016;8(11):7238-7247.

26. Kostura L, Kraitchman DL, Mackay AM, Pittenger MF, Bulte JW. Feridex labeling of mesenchymal stem cells inhibits chondrogenesis but not adipogenesis or osteogenesis. NMR Biomed. 2004;17(7): 513-517.

27. Mishra SK, Kumar BS, Khushu S, Tripathi RP, Gangenahalli G. Increased transverse relaxivity in ultrasmall superparamagnetic iron oxide nanoparticles used as MRI contrast agent for biomedical imaging. Contrast Media Mol Imaging. Epub 2016 May 27.
28. Raynal I, Prigent P, Peyramaure S, Najid A, Rebuzzi C, Corot C. Macrophage endocytosis of superparamagnetic iron oxide nanoparticles: mechanisms and comparison of ferumoxides and ferumoxtran-10. Invest Radiol. 2004;39(1):56-63.

29. Terrovitis J, Stuber M, Youssef A, et al. Magnetic resonance imaging overestimates ferumoxide-labeled stem cell survival after transplantation in the heart. Circulation. 2008;117(12):1555-1562.

30. Boden J, Lassance-Soares RM, Wang H, et al. Vascular regeneration in ischemic hindlimb by adeno-associated virus expressing conditionally silenced vascular endothelial growth factor. J Am Heart Assoc. 2016;5(6): $\mathrm{e} 001815$. 


\section{Supplementary material}
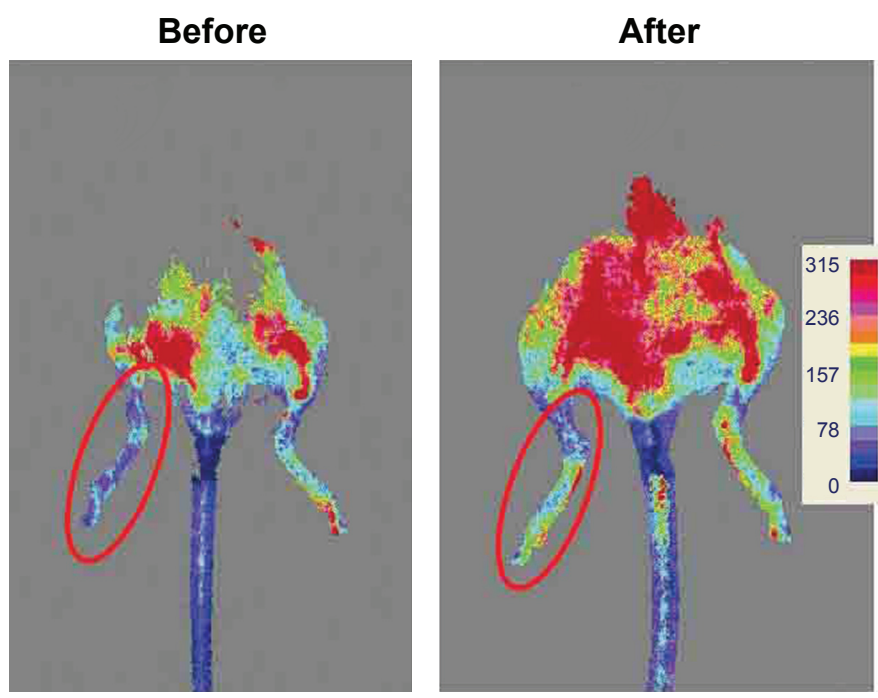

Figure SI Representative color laser Doppler images of superficial blood flow in lower ischemic limbs before and after treatment.

\section{Publish your work in this journal}

The International Journal of Nanomedicine is an international, peerreviewed journal focusing on the application of nanotechnology in diagnostics, therapeutics, and drug delivery systems throughou the biomedical field. This journal is indexed on PubMed Central,

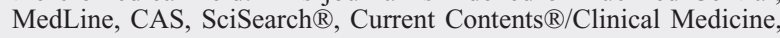

Journal Citation Reports/Science Edition, EMBase, Scopus and the Elsevier Bibliographic databases. The manuscript management system is completely online and includes a very quick and fair peer-review system, which is all easy to use. Visit http://www.dovepress.com/ testimonials.php to read real quotes from published authors. 